\title{
Comment on "Off-diagonal Long-range Order in Bose Liquids: Irrotational Flow and Quantization of Circulation"
}

\author{
$\mathrm{Yu} \mathrm{Sh} *$ \\ Cavendish Laboratory, University of Cambridge, Cambridge CB3 OHE, United Kingdom
}

\begin{abstract}
In the context of an application to superfluidity, it is elaborated how to do quantum mechanics of a system with a rotational velocity. Especially, in both the laboratory frame and the non-inertial corotating frame, the canonical momentum, which corresponds to the quantum mechanical momentum operator, contains a part due to the rotational velocity.
\end{abstract}

PACS numbers: 03.75.Fi, 67.40.-w, 74.25.-q

A recent Letter [1] claimed to have derived irrotational superflow or circulation quantization of a superfluid merely from the existence of off-diagonal long-range order (ODLRO). The derivation was based on an incorrect Hamiltonian.

In the beginning of that discussion, it is written: "Let us consider a bucket of Bose liquid composed of $N$ homogeneous interacting spinless particles, rotated with a constant angular velocity $\Omega$ " [1]. This clearly indicates that $\mathbf{v}_{s}=\boldsymbol{\Omega} \times \mathbf{r}$ is the fixed rotation velocity of the bucket, not the superfluid velocity. $\mathbf{v}_{s}$ and $\boldsymbol{\Omega}$ are both physical quantities, and one has no "gauge freedom" to add an additional term $\nabla \theta(\mathbf{r})$ in $\mathbf{v}_{s}$, as appearing in Eq. (2) of [1]. With this understanding, the conclusion that $\boldsymbol{\Omega}$ vanishes is, of course, absurd. In fact, for a superfluid in a rotating bucket, it is the Hamiltonian in the corotating frame that determines the equilibrium statistical mechanics 2, 3]. Instead of Eq. (1) of [1], the relevant Hamiltonian is $\sum_{j}\left[\frac{\left(\mathbf{p}_{j}-m \boldsymbol{\Omega} \times \mathbf{r}_{j}\right)^{2}}{2 m}-\frac{1}{2} m\left(\boldsymbol{\Omega} \times \mathbf{r}_{j}\right)^{2}\right]+$ $\frac{1}{2} \sum_{j \neq l} V\left(r_{j l}\right)$, where $\mathbf{p}_{j}=m\left(\mathbf{v}_{j}+\boldsymbol{\Omega} \times \mathbf{r}_{j}\right)$ is the canonical momentum and is then replaced as $-i \hbar \nabla_{j}, \mathbf{v}_{j}, \mathbf{r}_{j}$ and $\mathbf{p}_{j}$ are relative to the reference frame co-rotating with $\boldsymbol{\Omega} 2,3,4,[5]$. This Hamiltonian obviously lacks the invariance under an $\boldsymbol{\Omega} \times \mathbf{r}$-dependent re-gauged translation underlying the argument employed later in [1].

In their correspondence, the authors have claimed that they were not considering superfluid in a rotating bucket, instead, $\mathbf{v}_{s}=\boldsymbol{\Omega} \times \mathbf{r}+\nabla \theta$ was a "drift velocity" assumed to be a sum of an irrotational part $\nabla \theta$ and a rotational part $\boldsymbol{\Omega} \times \mathbf{r}$. Under this interpretation, even though the serious question about the physical meaning of this "drift velocity" is side-stepped, the Hamiltonian in [1] is still incorrect. In fact, whenever a certain "drift velocity" contains a rotational part $\boldsymbol{\Omega} \times \mathbf{r}$, the canonical momentum relative to the non-inertial frame co-moving with this "drift velocity" is $\mathbf{p}_{j}=m\left(\mathbf{v}_{j}+\boldsymbol{\Omega} \times \mathbf{r}_{j}\right)$, and thus in the laboratory frame, the kinetic part of the Hamiltonian $\left(\mathbf{p}_{j}+m \nabla \theta\right)^{2} / 2 m$ [4], in contrast with Eq. (1) of [1]. It is very elementary knowledge that it is canonical momentum that corresponds to momentum operator in quantum mechanics [4, 5]. Moreover, in [1], it is very mysterious why the assumed "drift velocity" suddenly became the superfluid velocity after the the rotational part was "shown" to vanish.

If the authors wish to adopt a reductio ad absurdam by showing that ODLRO is incompatible with the assumption that the superflow has a non-vanishing curl, i.e. the superfluid velocity is rotational, then the only valid interpretation is that $\mathbf{v}_{s}$ represents the superfluid velocity, first presumed to has a curl $2 \boldsymbol{\Omega}$; the purpose is then to show $\boldsymbol{\Omega}$ has to vanish under ODLRO. If we use a subscript 0 to represent the laboratory frame, then the assumption is $\frac{1}{2} \nabla_{0} \times \mathbf{v}_{s}=\boldsymbol{\Omega}$. With this interpretation, and if $\boldsymbol{\Omega}$ is assumed to be position-independent, one can write $\mathbf{v}_{s}=\boldsymbol{\Omega} \times \mathbf{r}_{0}+\nabla_{0} \theta\left(\boldsymbol{\Omega}, \mathbf{r}_{0}\right)$. In terms of the position vector $\mathbf{r}$ relative to the condensate, which moves with $\mathbf{v}_{s}$, the expression of $\mathbf{v}_{s}$ becomes very complicated. In general, Eq. (2) in [1] is valid only if $\mathbf{r}$ and thus $\mathbf{p}_{j}$ in [1] are relative to the rest frame. But this understanding cannot be consistent with the Hamiltonian in [1]. In fact, the Hamiltonian in the rest frame, in our notations, is simply $\sum_{j} \frac{\mathbf{p}_{j_{0}}{ }^{2}}{2 m}+\frac{1}{2} \sum_{i \neq l} V\left(r_{j l}\right)$, where $\mathbf{p}_{j_{0}}=-i \hbar \nabla_{j_{0}}$, instead of Eq. (1) of [1]. If one subsequently obtains (1) by a "gauge" transformation, then $m \mathbf{v}_{s} / \hbar$ is only the gradient of the phase factor, and $\mathbf{v}_{s}$ cannot be the superfluid velocity if it is presumed to be rotational as in (2) (cf. the preceding paragraph). On the other hand, it appears that $\mathbf{p}_{j}$ in [1] are relative to the condensate and that the authors wish to give the Hamiltonian in the rest frame expressed in terms of the momenta relative to the condensate. With this interpretation, however, both Eq. (2) in [1] and the expression $\boldsymbol{\Omega}=\frac{1}{2} \nabla \times \mathbf{v}_{s}$ below the equation are not valid generically under the presumption that the curl of the superfluid velocity is $\boldsymbol{\Omega}$ in the laboratory frame. Therefore the Hamiltonian in [1] is still incorrect.

It appears that the authors do not know that "irrotational superflow " just means that the curl of the superfluid velocity vanishes, in the laboratory frame, and that the purpose they had set for their Letter was just to show that with ODLRO, the superfluid velocity is of the form $\nabla \theta$, i.e. its curl vanishes. Moreover, they ignore the well-known fundamental difference between rotation and translation [2, 3, 4, 5]. Note that in Sec. 5.5 (pp. $75-76$ ) of Ref. [3], it is a uniform (as italicized there) translation of a superfluid that is considered.

I thank Nigel Cooper for useful discussions. 
* Electronic address: ys219@phy.cam.ac.uk

[1] G. Su and M. Suzuki, Phys. Rev. Lett. 86, 2708 (2001).

[2] L. D. Landau and E. M. Lifshitz, Statistical Mechanics (Pergamon Press, Oxford, 1976).
[3] P. Nozières and D. Pines The Theory of Quantum Liquids, Vol. 2 (Addison-Wesley, Redwood City, 1990).

[4] L. D. Landau and E. M. Lifshitz, Mechanics (Pergamon Press, Oxford, 1976), Sec. 39.

[5] L. D. Landau and E. M. Lifshitz, Quantum Mechanics (Pergamon Press, Oxford, 1976). 